\title{
MATHEMATICAL VISUALIZATION, MANIPULATIVES AND GEOMETRIC PROBLEM SOLVING: A CASE OF STUDY
}

\author{
Caterina Cumino \\ Department of Mathematical Sciences “G. L. Lagrange” (DISMA), Politecnico di Torino (Italy) \\ National Group for Algebraic and Geometric Structures \\ and their applications (GNSAGA-INDAM)
}

\begin{abstract}
The role of mathematical visualization in problem solving process and the incidence of material tools on apprehension of mathematical concepts have been subjects of intensive research for a long time.

In the present paper we analyze the use of a manipulative in solving a space Geometry problem, providing a critical account of its theoretical framework and its effectiveness in various teaching contexts.

Previous researches (Leikin, Stylianou, \& Silver, 2005) examined students' use of visual representations, across various ages and mathematical education levels, proposing a challenging task to the study participants: describe the net of a truncated right cylinder. Given the visual nature of the problem, any solution strategy required a translation between $3 \mathrm{D}$ and 2D representations, regardless of the more or less advanced mathematical knowledge. In the behavior of the successful visualizers, similarities were identified, such as selection of what needs to be visualized and transfer of visual images in a "symbol system", see Nemirovsky (1994); while both young and older low-achieving students reported difficulties in isolating object's relevant characteristics.

About the same problem, a physical manipulative was designed (Cumino, Spreafico, \& Zich, 2017) in a dissemination context where Geometry was a tool for understanding architectural shapes, under the need of quickly and correctly communicating the solution, avoiding mathematical formalizations: It shows a right cylinder truncated by oblique planes of various inclination and allows to obtain its net by a simple unwrap.

Currently, this manipulative is being tested at the university level, on first year students of the bachelor program in Architecture and at the middle school level, on second year students of a Technology course; in both teaching contexts, different for age and levels of mathematical knowledge, it is proving useful in making students able to elaborate individual solving strategies, to set up the problem correctly and to improve its translation in symbolic language for the holders of advanced mathematical tools, speeding up teachers' interventions.
\end{abstract}

Keywords: Visualization, geometric problem solving, physical manipulative, mathematical teaching strategies.

\section{Introduction and literature review}

Despite 3D Geometry is recognized as a way to develop spatial awareness and 3D thinking is suggested to be essential to creative thought (Presmeg, 2006), in school curricula Geometry is still being reduced in favor of numeracy skills, although by the end of the last century many scholars had begun to oppose this trend (Mammana \& Villani, 1998).

Even in author's teaching experience, a growing number of students accessing engineering or architecture studies encounter difficulties in dealing with 3D geometric problem solving, due to lack of practice in spatial reasoning and in those simple visual aspects of Geometry, which are at the basis of most STEM disciplines.

Therefore, the need is felt to improve teaching strategies at every school level, relying on investigations about the nature of growth in students' insight and mathematical competence into 3D Geometry.

In the last decades a consistent amount of research was focused on students' 3D geometric abilities and on their visual activities, see e.g. Presmeg, (2006). Mathematics indeed relies heavily on visualization and mathematical reasoning is part of a visualization process (Duval, 1999). Some scholars explored and 
coded the nature of students' reasoning about geometric solids (Lawrie, Pegg \& Gutierrez, 2000; Pittalis, Mousoulides, \& Christou, 2009; Stylianou \& Pitta-Pantazi, 2002) by examining and characterizing visual elements in the problem solving of persons with varying levels of mathematical knowledge and experience and their difficulties in finding relationships between the various parts of a solid; others, (Meng, 2012), investigated if achievements in 3D Geometry could be enhanced through phase-based instruction, in the sense of van Hiele (1986), using material tools (manipulatives).

Much of the research focused on spatial transformation tasks and on the abilities to translate between 3D solids and their 2D representations; a way in which these abilities can be observed is through recognition and construction of nets of solids (Lawrie et al. 2000; Stylianou, Leikin \& Silver, 1999; Cohen, 2003), that is the 2-D shape, sometimes called development, obtained by "unfolding" on a plane the whole surface of the solid or part of it. Visualizing solids nets, in particular nets of curved solids such as cones and cylinders, requires manipulation of visual imagery and transformation of one visual image to another. In their exploration of expert and novice visualization practices, Stylianou and Silver (2004) stated that novices produce visualizations, but they generally lack the necessary procedural knowledge that would allow them to use visual representations efficiently.

Instruction might help students develop awareness, analysis and connections between visual representations. Studies on using material objects in mathematical education (Sarama, 2016) generally indicates that physical representations play an important role at all school levels and that they can help students to improve not only learning processes of mathematical ideas but also problem-solving settings, although teacher guidance is required to ensure understanding and meaningful learning.

The present paper aims to provide a theoretical framework for a particular manipulative which has proved to be useful in a geometric problem-solving context at different school levels and to motivate its effectiveness, drawing on the previous researches.

\section{The case of study}

To investigate characteristics of visual representations underlying successful problem-solving, across students' ages and levels of mathematical knowledge, Stylianou et al. (1999) proposed the following mathematical task: given a right circular cylinder intersected by an oblique plane with respect to the cylinder axis, describe the truncated cylinder's net (see Figure 1 below).

Figure 1. Truncated cylinder: development of the lateral surface (elaborated from Leikin et al. (2005), p. 10).

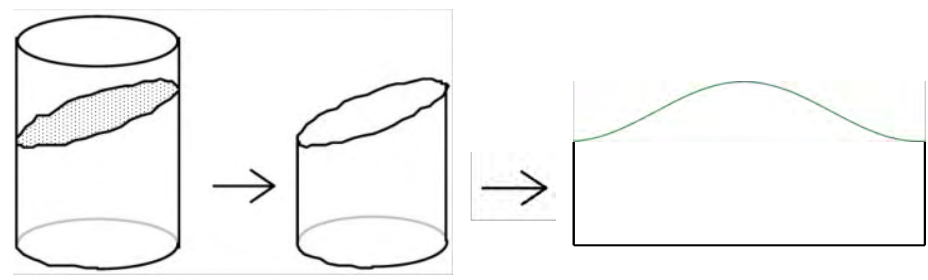

The problem appeared both challenging and nonroutine to subjects from different age groups (students from secondary school to university) and could be solved by everyone from an intuitive or a formal mathematical point of view. Formal solution involves sophisticated graphical construction (see Figure 2 below) or symbolic representation of the 3D and 2D figures and the ability to form connections between different symbolic representations. In particular, to describe the shape of the net's upper curve and formally prove that it is actually a sine wave, some advanced mathematical tools are necessary, such as the knowledge of trigonometric concepts and basics of Analytic Geometry; on the other hand, this problem could be approached intuitively yet rigorously by a student that does not have formal geometry or algebra knowledge.

Given the visual nature of the problem, any solution strategy requires a translation of a pictorial representation of the $3 \mathrm{D}$ truncated cylinder into its $2 \mathrm{D}$ net representation, regardless of the more or less advanced mathematical knowledge. 
Figure 2. Truncated cylinder's net: technical drawing.

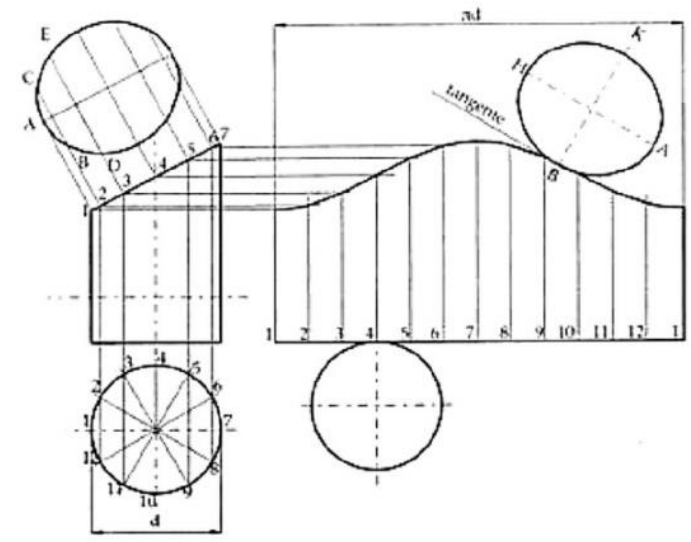

In the behavior of the successful visualizers Leikin et al. (2005) identified some re-occurring patterns, such as selection of what needs to be visualized and the transfer of visual images in a "symbol system" (in the sense of Nemirovsky, 1994), namely the mathematization of objects; while both younger and older low-achieving students reported difficulties in isolating objects relevant characteristics.

To approach the same problem, a physical manipulative was created (Cumino \& al., 2017) during the design of a set of guided tours in a Cultural Heritage context, involving people of different age and cultural background; the purpose of the project was to let visitors discover architectural shapes of an historical complex, using Geometry as a tool for understanding them; among the topics, it was planned to introduce typologies of vaults generated by intersections of cylinders, such as groin vaults and cloister vaults; various educational materials were proposed to enhance visitors observation, including origami like paper models; small groups of secondary school students of different age were involved in dissemination mathematical workshops to understand the geometric concepts underlying those models, in particular to study intersections of cylinders with a plane and their developments: thus, there was a need of conveying in an intuitive, quick yet correct way the solution of the truncated cylinder problem, avoiding mathematical formalizations.

The manipulative consists of a plank of rigid material (cardboard or plexiglas) with holes of various shapes and a right circular cylinder of the same material; after having wrapped a sheet of paper around the cylinder and having inserted the cylinder so wrapped in the different circular and elliptical holes, it is thus possible to draw the plane-cylinder intersection curve: then, unfolding the sheet of paper allows to perceive by direct experimentation the top profile of the net and to realize how it varies when the plane changes inclination.

Figure 3. Studying cylinder plane sections and their development.

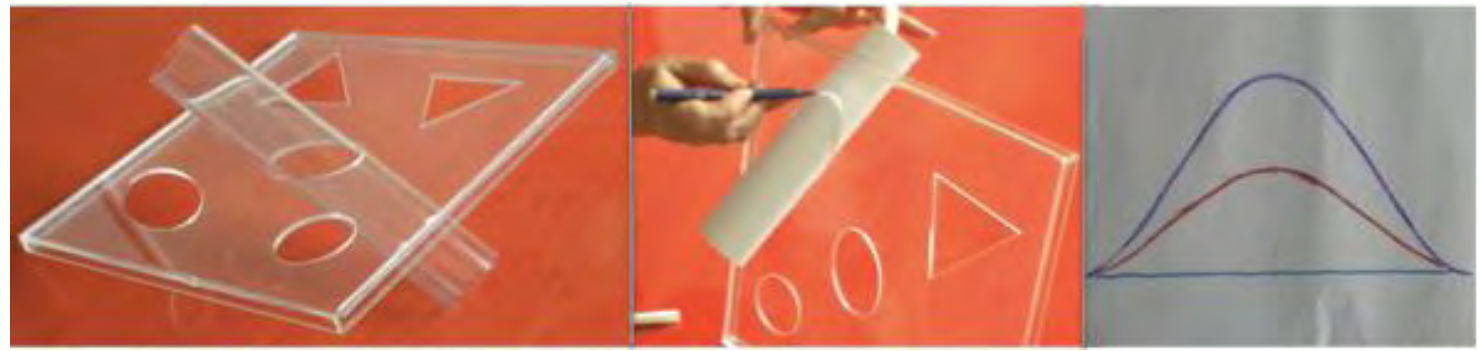

In the first feasibility checks on pilot groups of middle and high school students, this educational artifact proved to be useful to make people elaborate individual solving strategies and to set up the problem correctly.

Subsequently, this experience was applied in other contexts. Currently, this manipulative is being tested at university level, on first year students of the bachelor program in Architecture in occasion of interdisciplinary activities about vaulted systems between the courses of Calculus, Architectural Drawing and Survey Laboratory (Cumino \& al., 2019); in this situation, learners have tools of trigonometry and are acquiring basics of analytic geometry and graphic language: the use of the manipulative seems to quickly lead them to a correct problem setting and to improve translations between different representations, speeding up teachers' interventions. 
A similar investigation is planned in a completely different environment, after a preliminary analysis phase: namely on second year students of a Technology course, at middle school level, where one of the objectives of the educational program is to observe architectural shapes in the surrounding world with particular attention to examples of roofing and vaulted systems and learners are asked to construct simple paper models of buildings, so they have to consider nets of solids. To approach the truncated cylinder problem these students initially behave like the older ones: as remarked by Leikin et al. (2005), they try to identify those properties of the truncated cylinder which may be important to find the net, but a lack of mathematical knowledge and spatial reasoning experience prevents them from going beyond their initial observation of the object as a whole. The manipulative in this case allows them an intuitive but rigorous approach, letting them overcome conceptual obstacles.

\section{Conclusion}

The purpose of the present paper was to investigate the reason why a specific educational tool works with students having different mathematical literacy. The study is part of a research program dedicated to the dissemination of Mathematics, with the aim of presenting topics of 3D Geometry at any scholastic or university level as rigorously as possible, stimulating an inclusive perceptive-motor learning, proposing calculation or graphic construction only when necessary and in a functional manner, depending on the students' background.

\section{References}

Cohen, N. (2003). Curved Solid Nets. In N. Pateman, B. J. Dougherty, \& J. Zillox (Eds.), Proceedings of the 27th $24^{\text {th }}$ annual conference of the IGPME. Honolulu, USA, July 13-18, 2003. Vol. 2 (pp. 229-236). Honolulu: PME.

Cumino, C., Spreafico, M.L., \& Zich (2017). "The King and the Origami”, educational tours at the royal Residence of Venaria Reale (Italy) to observe, analyze and model Architecture through Mathematics, Proceedings of the 16th Conference on Applied Mathematics APLIMAT 2017, February 1-2, 2017, Bratislava, Slovacchia (pp. 428-439). Bratislava: Vydavatel'stvo Spektrum STU.

Cumino, C., Pavignano, M., Spreafico, M.L., and Zich, U. (2019). Teaching Geometry and Surfaces Evaluation Through Graphic Representation and Dynamic Paper Models. In L. Cocchiarella (Ed.), 2018 - Proceedings of the 18th International Conference on Geometry and Graphics, Aug. 3-7, Milan, Italy (pp. 1523-1532). Cham: Springer.

Duval, R. (1999), Representation, Vision and Visualization: Cognitive Functions in Mathematical Thinking Basic Issues for Learning. In: F. Hitt, M. Santos, (Eds.). Proceedings of the annual meeting of the North American Chapter of the IGPME. Cuernavaca, Morelos, Mexico, October 23-26, 1999. Vol. 1 (pp. 3-26). Columbus: Eric.

Lawrie, C., Pegg, J. \& Gutierrez, A. (2000). Coding the nature of thinking displayed in responses on nets of solids. In T. Nakahara \& M. Koyama (Eds.), Proceedings of the $24^{\text {th }}$ annual conference of the IGPME. Hiroshima, Japan, July 23-27, 2000. Vol. 3 (pp. 215-222). Hiroshima: Eric.

Leikin, R., Stylianou, \& D., Silver, E. (2005). Visualization and mathematical knowledge: Drawing the net of a truncated cylinder. Mediterranean Journal for Research in Mathematical Education, 4,1, pp. 1-39.

Mammana, C., \& Villani, V. (Eds.). (1998). Perspectives on the Teaching of Geometry for the 21th Century: an ICMI study. Dodrecht, the Netherlands: Kluwer.

Meng, C. \& Idris, N. (2012). Enhancing Students' Geometric Thinking and Achievement in Solid Geometry. Journal of Mathematics Education, Vol. 5, No. 1, pp. 15-33.

Nemirovsky, R. (1994). On ways of symbolizing: The case of Laura and velocity sign. The Journal of Mathematical Behavior 13, 389-422.

Pittalis, M., Mousoulides, N. \& Constantinos, C. (2009). Students' 3D Geometry Thinking Profiles. Proceedings of CERME 6, Lyon, France, January 28th-February $1^{\text {st }}$, 2009. (pp. 816-825). Lyon: INRP.

Presmeg, N. (2006). Research on visualization in learning and teaching mathematics. In A. Gutierrez \& P. Boero (Eds.), Handbook of Research on the PME: Past, Present and Future (pp. 205-236). Rotterdam: Sense Publishers.

Sarama, J., \& Clements, D. H. (2016). Physical and Virtual Manipulatives: What Is "Concrete"? In P. S. Moyer-Packenham (Ed.), International Perspectives on Teaching and Learning Mathematics with Virtual Manipulatives (Vol. 7, pp. 71-93). Cham: Springer International Publishing. 
Stylianou, D., \& Silver, E. (2004). The Role of Visual Representations in Advanced Mathematical Problem Solving: An Examination of Expert-Novice Similarities and Differences, Mathematical Thinking and Learning, 6, 4, pp. 353-387.

Stylianou, D., Pitta-Pantazi, D. (2002). Visualization and High Achievement in Mathematics: A Critical Look at Successful Visualization Strategies. In F. Hitt (Ed.), Representation and mathematics visualization, (pp. 31-45). Mexico: PME-NA and Cinvestav - IPN.

Stylianou, D., Leikin, R. \& Silver, E. (1999). Exploring students' solution strategies in solving a spatial visualization problem involving nets. In O. Zaslavsky (Ed.), Proceedings of the 23rd annual conference of the IGPME. Haifa, Israel, July 25-30, 1999. Vol. 4 (pp. 241-248). Haifa: Technion Printing Center.

van Hiele, P. M. (1986). Structure and Insight. A Theory of Mathematics Education. Florida, USA: Academic Press. 